\title{
ORIGINAL ARTICLE Changes in the structural and material properties of the tibia in patients with spinal cord injury
}

\author{
ID McCarthy ${ }^{1}, \mathrm{Z}_{\text {Bloomer }}{ }^{1}, \mathrm{~A}_{\text {Gall }}^{2}, \mathrm{R} \mathrm{Keen}^{1}$ and M Ferguson-Pell ${ }^{3}$
}

Study design: A cross-sectional study.

Objectives: To measure the change of structural and material properties at different sites of the tibia in spinal cord-injured patients using peripheral quantitative computerised tomography (pQCT).

Setting: Orthopaedic research centre (UK).

Methods: Thirty-one subjects were measured-eight with acute spinal cord injury (SCI), nine with chronic SCl and fourteen ablebodied controls. pQCT scans were performed at 2\% (proximal), 34\% (diaphyseal) and 96\% (distal) along the tibia from the tibial plateau. Structural measures of bone were calculated, and volumetric bone mineral density (vBMD) was also measured at all three levels. Muscle cross-sectional area was measured at the diaphyseal level.

Results: Structurally, there were changes in the cortical bone; in the diaphysis, the shape of the cross-section changed to offer less resistance to AP bending, and the cross-sectional area of the cortical shell decreased both proximally and distally. There were corresponding changes in VBMD in the anterior aspect of the cortical diaphysis, as well as proximal and distal trabecular bone. Changes in muscle occurred more rapidly than changes in bone.

Conclusion: There were clear changes of both structure and material at all three levels of the tibia in chronic SCl patients. These changes were consistent with specific adaptations to reduced local mechanical loading conditions. To assess fracture risk in SCI and also to monitor the effect of therapeutic interventions, the structure of the bone should be considered in addition to trabecular bone mineral density.

Spinal Cord (2012) 50, 333-337; doi:10.1038/sc.2011.143; published online 29 November 2011

Keywords: spinal cord injuries_complications; bone density; biomechanics

\section{INTRODUCTION}

Patients with spinal cord injury (SCI) lose bone below the level of their injury. ${ }^{1,2}$ The rate of bone loss is rapid in the first 2 years, but a steady state at a reduced level is eventually reached. The decrease in bone mass is associated with an increase in fracture risk, with SCI patients having approximately a twofold increase in fracture risk compared with controls. As a result, there have been investigations of both pharmacological and non-pharmacological methods of reducing bone loss and fracture risk. ${ }^{3,4}$

Both dual-energy X-ray absorptiometry (DXA) and peripheral quantitative computerised tomography (pQCT) have been used to investigate bone changes after SCI. ${ }^{5-7}$ Although there are large databases of DXA values in the lumbar spine, proximal femur and distal radius, most fractures in SCI patients occur in the distal femur or proximal tibia; these are not sites normally scanned by DXA and therefore there is no large database of controls. pQCT data has been reported for the distal tibia, ${ }^{6,8}$ but not together with data on proximal tibia, which is a more common site for fractures in SCI patients. It has been proposed that bone mineral should be measured round the knee, ${ }^{3}$ and Rittweger et al. measured four sites around the knee, two in the distal femur and two in the proximal tibia. ${ }^{9}$

At sites where it is possible to use pQCT, there are significant advantages of using this technique. Firstly, it measures true volumetric bone mineral density (vBMD). Several studies have shown a relation- ship between bone density and both elastic modulus and ultimate strength. Thus vBMD is an index of the material properties of the bone. In addition, pQCT can provide information on structural parameters of the bone that contribute to the mechanical strength of the whole bone. Cross-sectional area is relevant in the resistance of bone to axial compressive loads (relevant at the proximal and distal ends); cross-sectional moment of inertia determines the ability of the bone to resist bending, which is the most relevant structural parameter for the diaphysis.

Therefore, the aim of the present study was to use pQCT to compare changes of both the structure and vBMD in the tibiae of SCI patients, at the proximal metaphysis, the diaphyseal cortex and the distal metaphysis of the tibia.

\section{METHODS}

Subjects

Seventeen SCI subjects (all male) were recruited from the London Spinal Cord Injuries Centre, Royal National Orthopaedic Hospital. These were classified as either acute (time since injury $<6$ months) or chronic (time since injury $>48$ months). Fourteen male age-matched ablebodied controls ( $\mathrm{AB}$ group) were also recruited. The study was approved by the joint Royal National Orthopaedic Hospital/UCL Institute of Orthopaedics and Musculoskeletal Science Research Ethics Committee. Written informed consent was obtained from all participants.

${ }^{1}$ UCL Institute of Orthopaedics and Musculoskeletal Science, Stanmore, UK; ${ }^{2}$ London Spinal Cord Injuries Centre, Royal National Orthopaedic Hospital, Stanmore, UK and ${ }^{3}$ Faculty of Rehabilitation Medicine, Corbett Hall, University of Alberta, Edmonton, Alberta, Canada

Correspondence: Dr ID McCarthy, UCL Institute of Orthopaedics and Musculoskeletal Science, Royal National Orthopaedic Hospital, Brockley Hill, Stanmore, Middlesex HA7 4LP, UK. E-mail: i.mccarthy@ucl.ac.uk

Received 12 May 2011; revised 13 October 2011; accepted 23 October 2011; published online 29 November 2011 


\section{Measurement}

Bone mineral density (BMD) measurements were performed using pQCT using an XCT 3000 scanner (Stratec, Pforzheim, Germany). Subjects lay supine on a bed, and were moved to the end of the bed so that one leg could be placed in the scanner, while the contralateral leg was supported beside the scanner. The length of the tibia was measured. The position of the patient adjusted so that the positioning beam in the scanner was just at the distal tip of the patella. A scout view was performed to identify the position of the tibial plateau and the start line was moved to coincide with the tibial plateau. Scans were performed at $2 \%$ (proximal trabecular bone) and 34\% along the length of the bone from the tibial plateau (diaphyseal cortex at a level that approximates to the maximal cross-sectional area of muscle). As the gantry of the scanner could not move the whole length of the tibia, the patient was then moved to measure the distal tibia. A scout view was performed to identify the position of the ankle, and then a scan was performed $4 \%$ along the length of the tibia from the ankle (96\% along the length of the bone from the tibial plateau). For practical reasons, scans of the distal tibia were not performed on acutely injured subjects.

\section{Analysis}

The following parameters were calculated from the scans: trabecular vBMD proximally and distally; cortical vBMD in the diaphyseal cortex; local vBMD in anterior, lateral, posterior and medial regions of interest in the diaphyseal cortex; cortical bone area in the diaphyseal cortex and in the proximal and distal cortical shells; second moment of area about the $x$ and $y$ axes for diaphyseal cortex (Ixx and Iyy); circularity estimated from the ratio Ixx/Iyy; cross-sectional area of muscle. Axes for the calculations of Ixx and Iyy, and the regions of interest for local vBMD are shown in Figure 1. Statistical analysis of group differences was performed by one-way ANOVA.

\section{RESULTS}

Basic information on the patients is shown in Table 1. The mean age of the patients was $33.0 \pm 12.2$ and $32.1 \pm 8.9$ years in the acute and chronic SCI groups, respectively, and the mean age of the AB controls was $36.6 \pm 9.1$ years. The mean times since injury were $4.0 \pm 2.1$ and $77.1 \pm 33.4$ months in the acute SCI and chronic SCI groups, respectively. Examples of scans at the three different levels are shown in Figure 2, for a control subject and a chronic SCI subject. Qualitatively, it can be appreciated that the trabecular bone is very different in the SCI patients; in contrast, differences in the diaphyseal cortex are not immediately apparent from basic inspection, but become apparent in quantitative analysis.

\section{Material properties}

The mean proximal and distal trabecular BMDs are shown in Table 2. There is a clear trend of decreasing BMD from controls to acutely injured patients to chronically injured patients. ANOVA shows a statistically significant difference between the groups, but post-hoc analysis shows no difference between the controls and acutely injured patients, whereas both these groups are significantly different from the chronic SCI group. In the proximal tibia, trabecular BMD in chronic SCI subjects was reduced by almost $40 \%$ compared with $\mathrm{AB}$ controls, from $212.8 \pm 51.1$ to $131.5 \pm 51.1 \mathrm{mg} \mathrm{cm}^{-3}$ (mean \pm s.d., $P=0.013$ ), but in the distal tibia trabecular BMD was decreased by more than $60 \%$, from $260.8 \pm 48.2$ to $99.2 \pm 43.8 \mathrm{mg} \mathrm{cm}^{-3}(P<0.001)$.

Values for cortical BMD in the diaphysis are shown in Table 3. Although there is a trend of decreasing density from control through to the chronic SCI group, these differences are not statistically

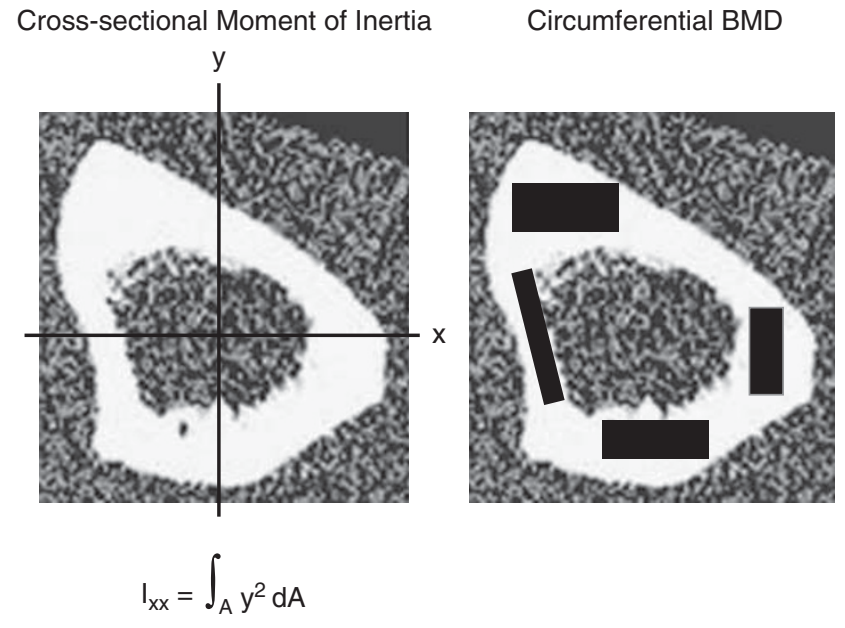

Figure 1 Illustration of the axes for calculation of cross-sectional moment of inertia, and the anterior, posterior, lateral and medial regions of interest that were used for calculation of local BMD.

Table 1 Details of $\mathrm{SCl}$ subjects' age, time since injury and AIS classification

\begin{tabular}{ccc}
\hline Age (years) & Time since injury (months) & AlS \\
\hline Acute & & \\
32 & 6 & T A \\
51 & 5 & T A \\
25 & 6 & T A \\
52 & 2 & T A \\
24 & 6 & C A \\
34 & 4 & T A \\
21 & 1 & T A \\
25 & 2 & T A \\
& & \\
Chronic & & T A \\
25 & 55 & T A \\
20 & 49 & T A \\
43 & 51 & T A \\
43 & 55 & C A \\
27 & 74 & T A \\
24 & 84 & T A \\
38 & 72 & C A \\
29 & 108 & T A \\
40 & 151 &
\end{tabular}

Abbreviations: A, complete; AIS, American Spinal Injury Association Impairment Scale; C, cervical; SCl, spinal cord injury; T, thoracic.

significant $(P=0.105)$. Although there is no difference in overall cortical BMD, there are interesting variations in BMD circumferentially, when defining regions of interest in the anterior, posterior, medial and lateral quadrants. These are shown in Figure 3. It can be seen that there are small differences between the quadrants in both SCI and control subjects. For all three groups, the variation in vBMD is statistically significant $(P=0.006, P=0.001$, and $P=0.001$ for the $\mathrm{AB}$ group, acute SCI and chronic SCI, respectively), but the pattern of variation is different for the $\mathrm{AB}$ group and the two SCI groups. It can be seen that changes in cortical BMD in SCI subjects occur locally, and that BMD in the anterior aspect of the tibia is significantly reduced. 
Control

Chronic SCI

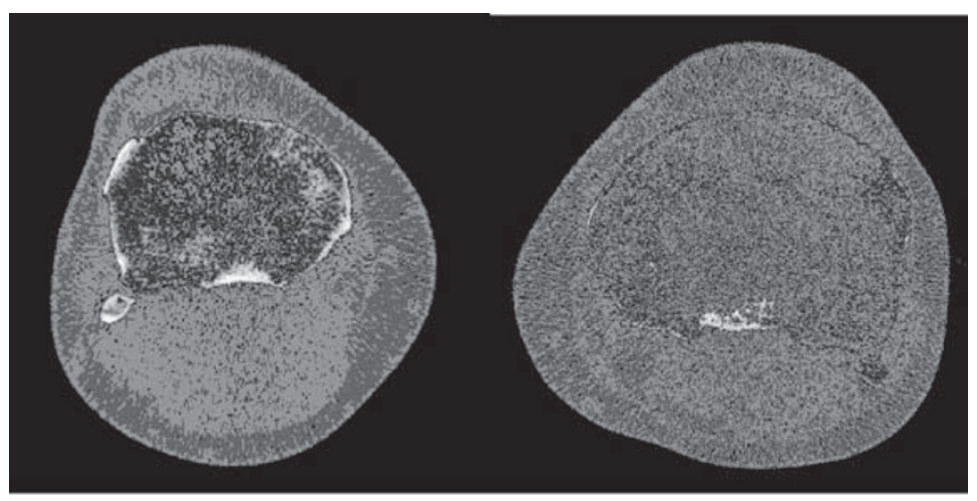

Proximal

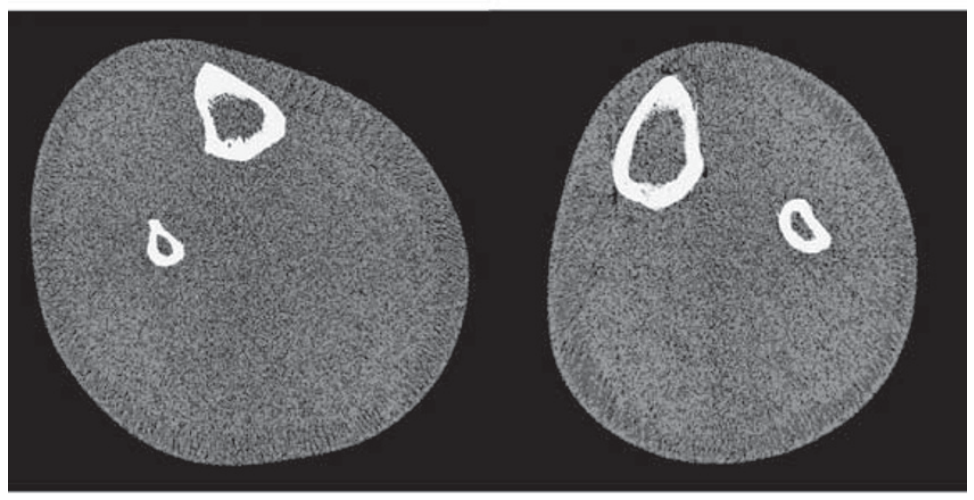

Diaphyseal

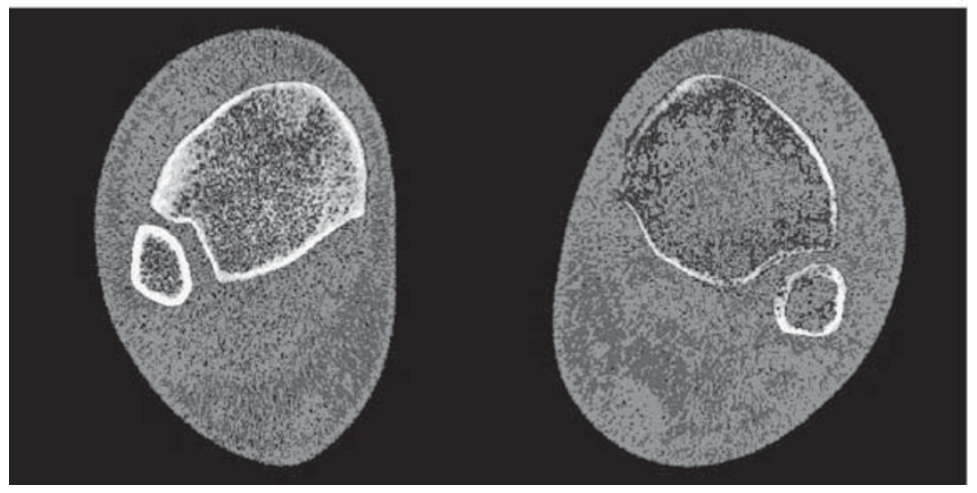

Distal

Figure 2 Examples of pQCT scans of the proximal tibia 2\% along the bone from the tibial plateau, in the diaphyseal cortex $34 \%$ along the bone, and the distal tibia, $96 \%$ along the bone; left, scans from a control subject, and right, scans from SCl patients. Images are not of the same scale.

Table 2 Proximal and distal trabecular BMD in AB controls, Acute $\mathrm{SCl}$ and chronic $\mathrm{SCl}$ subjects

\begin{tabular}{lcc}
\hline & $\begin{array}{c}\text { Proximal trabecular } \\
B M D\left(\mathrm{mgcm}^{-3}\right)\end{array}$ & $\begin{array}{c}\text { Distal trabecular } \\
B M D\left(\mathrm{mgcm}^{-3}\right)\end{array}$ \\
\hline AB & $212.8 \pm 48.9$ & $260.8 \pm 48.2$ \\
Acute $\mathrm{SCl}$ & $187.5 \pm 33.4$ & - \\
Chronic $\mathrm{SCl}$ & $131.5 \pm 51.1^{\mathrm{a}}$ & $99.2 \pm 43.8^{\mathrm{a}}$ \\
\hline
\end{tabular}

Abbreviations: $\mathrm{AB}$, able-bodied controls; $\mathrm{BMD}$, bone mineral density; $\mathrm{SCI}$, spinal cord injury. astatistically different from both $\mathrm{AB}$ controls and acute $\mathrm{SCl}(P<0.001)$.

\section{Structural properties}

Measurements of cortical bone cross-sectional area are shown for the proximal and distal cortical shells and diaphyseal cortex in Table 4. Using ANOVA, there are differences in the groups for
Table 3 Cortical vBMD for the entire slice of the diaphyseal cortex in $\mathrm{AB}$ controls, acute $\mathrm{SCl}$ and chronic $\mathrm{SCl}$ subjects

\begin{tabular}{lc}
\hline & Diaphyseal $B M D\left(\mathrm{mgcm}^{-3}\right)$ \\
\hline $\mathrm{AB}$ & $1155.7 \pm 22.0$ \\
Acute $\mathrm{SCl}$ & $1138.6 \pm 42.4$ \\
Chronic $\mathrm{SCl}$ & $1125.5 \pm 27.2$ \\
\hline Abbreviations: AB, able-bodied controls; SCl, spinal cord injury; $v B M D$, volumetric bone density.
\end{tabular}

proximal and diaphyseal bone ( $P=0.009$ and $P=0.001$, respectively) and post-hoc analysis showed differences between the chronic SCI group and both $\mathrm{AB}$ and acute SCI groups. For distal cortical bone, where no measurements were performed on the 


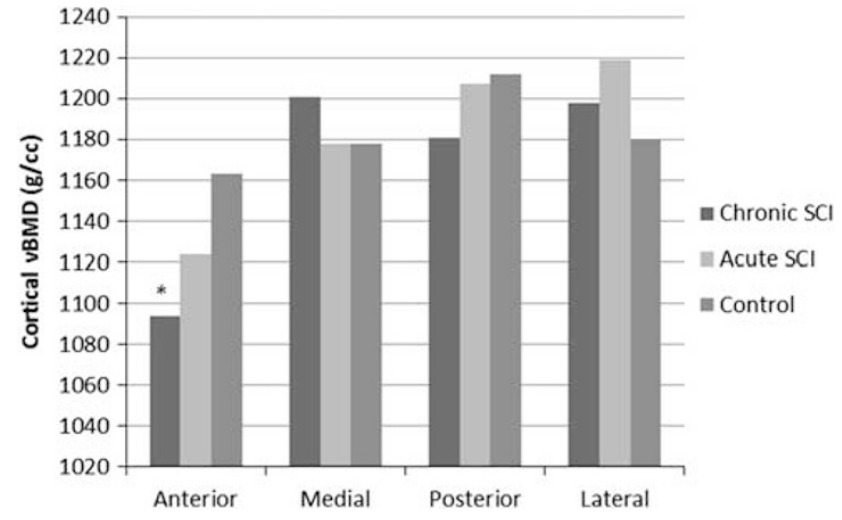

Figure 3 Distribution of cortical BMD between anterior, posterior, medial and lateral quadrants in acute $\mathrm{SCl}$, chronic $\mathrm{SCl}$ and control $\mathrm{AB}$ subjects. *Significantly different from anterior BMD in $A B$ controls $(P<0.001)$.

Table 4 Cross-sectional area of cortical bone in the proximal and distal cortical shells and diaphyseal cortex for AB controls, Acute $\mathrm{SCl}$ and Chronic SCl subjects

\begin{tabular}{lccc}
\hline & Proximal $\left(\mathrm{mm}^{2}\right)$ & Diaphyseal $\left(\mathrm{mm}^{2}\right)$ & Distal $\left(\mathrm{mm}^{2}\right)$ \\
\hline AB & $42.7 \pm 19.8$ & $374.8 \pm 34.6$ & $60.3 \pm 34.2$ \\
Acute SCl & $27.9 \pm 12.6$ & $368.0 \pm 42.1$ & - \\
Chronic SCl & $20.2 \pm 17.4$ & $297.9 \pm 30.7$ & $26.4 \pm 24.3$ \\
\hline
\end{tabular}

Abbreviation: $\mathrm{SCl}$, spinal cord injury.

Table 5 Cross-sectional moments of inertia for the tibial diaphysis, $34 \%$ along the length of the bone from the tibia plateau

\begin{tabular}{lccc}
\hline & Ixx $\left(\mathrm{mm}^{4}\right)$ & lyy $\left(\mathrm{mm}^{4}\right)$ & Circularity \\
\hline AB & $34289 \pm 10291$ & $23843 \pm 4891$ & $0.733 \pm 0.193$ \\
Acute SCI & $33270 \pm 12701$ & $24423 \pm 7442$ & $0.805 \pm 0.324$ \\
Chronic SCl & $22450 \pm 5267^{a}$ & $20166 \pm 3562$ & $0.912 \pm 0.300$ \\
\hline
\end{tabular}

Abbreviation: $\mathrm{SCl}$, spinal cord injury.

astatistically different from $\mathrm{AB}$ controls $(P<0.022)$.

acutely injured patients, there was a significant difference between $\mathrm{AB}$ and chronic SCI $(P=0.031)$. The measured decreases in area in the proximal and distal cortical shells were of the order of $50 \%$

Mean values of the two orthogonal cross-sectional moments of inertia (Ixx and Iyy) for the diaphyseal cortex are shown in Table 5. All values are reduced for the SCI groups, but this is only statistically significant for $\operatorname{Ixx}(P=0.019)$. Post-hoc analysis showed differences between the control group and the chronic SCI group only. Circularity was estimated from the ratio of Iyy/Ixx, which will equal 1 for a circular cross-section. Values for circularity are also shown in Table 5, although there is a trend towards greater circularity in the SCI patients, this was not statistically significant $(P=0.172)$.

\section{Muscle cross-sectional area}

Data for muscle cross-sectional area are shown in Table 6. Area was different between the groups $(P<0.001)$, but there were no differences
Table 6 Calf muscle cross-sectional areas for $\mathrm{AB}$, acute $\mathrm{SCl}$ and chronic $\mathrm{SCl}$ at the diaphyseal level

\begin{tabular}{lc}
\hline & Muscle cross-sectional area $\left(\mathrm{mm}^{2}\right)$ \\
\hline $\mathrm{AB}$ & $8617.7 \pm 1255.2$ \\
Acute SCl & $5695.4 \pm 855.5^{\mathrm{a}}$ \\
Chronic SCl & $5641.1 \pm 889.6^{\mathrm{a}}$ \\
\hline
\end{tabular}

Abbreviations: $\mathrm{AB}$, able-bodied controls; $\mathrm{SCl}$, spinal cord injury.

aStatistically different from $\mathrm{AB}$ controls $(P<0.001)$.

between either of the SCI groups, but both were significantly lower than the $\mathrm{AB}$ group.

\section{DISCUSSION}

In this study, we have used pQCT to investigate changes in the tibia of patients with SCI. The shape of the diaphysis changed to reflect reduced bending, and there were also changes in the thickness of the cortical shell proximally and distally. Changes in vBMD were observed locally within cortical bone, as well as in both proximal and distal trabecular bone. The advantage of pQCT over DXA is that pQCT can provide information about both structural and local material properties of bone being investigated. pQCT has been used to perform a structural analysis of the human tibia; ${ }^{10}$ scans were performed at $5 \%$ intervals along the length of the tibia in 20 healthy subjects. It was concluded that tibial anatomy is adapted to the mechanical stress distribution resulting from weight-bearing and normal activities of daily living. It would be difficult to perform such detailed measurements on SCI patients, as, due to spasms, it could be difficult for them to remain still for 19 serial scans. However, measurement at three sites provides interesting information, and shows that there are both structural and material changes in the tibiae of SCI patients. Although SCI subjects lose bone rapidly after injury, the decreases in vBMD we observed in the acute SCI group did not reach statistical significance, and statistical significance was only observed in the chronically injured subjects. Data for muscle, however, showed very large changes in the acute group, and there was no subsequent change in the muscle area.

Structurally, cortices become thinner, the moments of inertia decrease, and the bones tend to become more circular in cross-section. If detailed bone morphology is determined by mechanical loading, it makes sense that the diaphyseal cortex of tibiae in SCI patients should become more circular in the absence of significant mechanical loading. In control subjects, the bone in the diaphysis has greater resistance to AP bending than ML bending, presumably because of the action of the calf muscles inducing significantly more bending moment in the anteroposterior (AP) direction, compared with the mediolateral (ML) direction. In SCI subjects, the action of the calf muscles is significantly reduced or completely absent, and therefore the tibia does not need to resist bending in this direction. In the chronic SCI group, moments of inertia are almost equal in the two orthogonal directions, suggesting no preferential loading direction. Changes in cortical BMD also support this interpretation. There are small but significant differences in the cortical BMD of the SCI patients, and these occur in the anterior aspect of the tibiae. The morphology of the tibiae is changing so that the moment of inertia in the AP direction is reducing, and so the change in BMD in the anterior quadrant could be the result of increased remodelling in this region, in which there would be a higher proportion of partially mineralised osteons and increased porosity of the bone. The resolution of the pQCT scanner used in this study was not sufficient to detect porosity within cortical bone. It is interesting that changes were observed in the anterior 
quadrant of the tibia. From the position of the calf muscles one might expect the anterior quadrant of the tibia to be subject to tension during loading; measurement of strain on the human tibia has shown large tensile strains on the tibia during running, though the loading direction does depend on the activity. ${ }^{11}$ In experiments on fluid movement in bone, it has been shown that there is enhanced perfusion of the extracellular fluid space in bone in regions subjected to tension, ${ }^{12}$ which suggests a role for bone-fluid flow in the bone changes seen in the SCI subjects.

A significant decrease in the area of the cortical shell was seen in the chronic SCI group, but care must be taken when interpreting these values. In these regions the cortex is rather thin, and inaccuracies may result from the partial volume effect, in which voxels can either be detected as part of cortical bone despite not being filled completely by bone, or can be disregarded despite not being completely free of bone mineral. The beam width of the Stratec XCT 3000 is $2 \mathrm{~mm}$, and scans were performed with a pixel size of $0.3 \mathrm{~mm}$. In the diaphyseal cortex, the cortical thickness is around $4.3 \mathrm{~mm}$, many times the pixel size, and this reduces considerably the partial volume effect. In the proximal and distal cortical shells, the thickness of the cortical bone is only two or three pixels, giving uncertainty to the absolute values. However, the partial volume effect will tend to overestimate thickness, so there are clearly significant changes occurring in the proximal and distal shells, which are sites of fractures in SCI patients. Recently, it has been suggested that the thickness of the cortical shell in the femoral neck contributes more to the mechanical strength than the trabecular bone density. ${ }^{13}$ Results from the present paper suggest that the proximal and distal cortical shell in SCI patients should be studied in more detail.

Macroscopically, changes in the trabecular bone could be analysed as changes in a material property (that is, vBMD), although microscopically changes in trabecular bone could be regarded as structural, but the changes in trabecular number and thickness are not detectable with the resolution of the scanner. Changes in density were significantly higher in the distal metaphysis compared with the proximal metaphysis, though the observed changes in this paper were smaller than the $73 \%$ decrease in trabecular BMD reported elsewhere for the distal tibia. ${ }^{6}$ Treadmill walking has been shown to improve trabecular BMD in the distal tibia but not in the proximal tibia in a case report. ${ }^{8}$ The bone mineral content at the distal end is about $50 \%$ of that at the proximal ends, and as both proximal and distal metaphyses are loaded primarily in compression it is likely that strains in the distal tibia will be correspondingly larger, perhaps explaining the greater responsiveness of the distal bone to the absence of mechanical stimulus.

The study has investigated two distinct groups of SCI subjects: those with acute injuries ( $<6$ months) and those with chronic injuries ( $>48$ months). Most of the significant changes were seen in the chronic group. In the future, however, it would be interesting to extend this work to SCI subjects with a broader distribution of times since injury, so that the time course of these structural changes can be studied.

In summary, there were changes in both structural and material properties of the tibiae in SCI subjects. Cortices became thinner at all three levels; the moment of inertia in the diaphysis reduced in the AP direction, and this was accompanied by reduced BMD in the anterior quadrant of the bone. Trabecular BMD changed both proximally and distally, but the magnitude of change was greater distally, perhaps explained by greater strains occurring distally because of the smaller bone mineral content. We believe that these structural changes in bone are an important aspect of the increased fracture risk in SCI.

\section{CONFLICT OF INTEREST}

The authors declare no conflict of interest.

\section{ACKNOWLEDGEMENTS}

We are grateful to the Action Medical Research for funding this work.

1 Jiang S-D, Dai L-Y, Jiang L-S. Osteoporosis after spinal cord injury. Osteoporos Int 2006; 17: 180-192.

2 Zehnder $\mathrm{Y}$, Luthi M, Michel D, Knecht H, Perrelet R, Neto I et al. Long-term changes in bone metabolism, bone mineral density, quatitative ultrasound parameters, and fracture incidence after spinal cord injury: a cross-sectional study in 100 paraplegic men. Osteoporos Int 2004; 15: 180-189.

3 Biering-Sorensen F, Hansen B, Lee BSB. Non-pharmocological treatment and prevention of bone loss after spinal cord injury: a systematic review. Spinal Cord 2009; 47: 508-518.

4 Bubbear JS, Gall A, Middleton FRI, Ferguson-Pell M, Swaminathan R, Keen R. Early treatment with zolendronic acid prevents bone loss at the hip following acute spinal cord injury. Osteoporos Int 2011; 22: 271-279.

5 Bauman WA, Schwartz E, Song ISY, Kirshblum S, Cirnigliaro C, Morrison N et al. Dualenergy $\mathrm{X}$-ray absorptiometry overestimates bone mineral density of the lumbar spine in persons with spinal cord injury. Spinal Cord 2009; 47: 628-633.

6 Eser P, Frotzler A, Zehnder Y, Wick L, Knecht H, Denoth J et al. Relationship between the duration of paralysis and bone structure; a pQCT study of spinal cord injured individuals. Bone 2004; 34: 869-880.

7 Frotzler A, Berger M, Knecht H, Eser P. Bone steady-state is established at reduced bone strength after spinal cord injury: a longitudinal study using peripheral quantitative computed tomography. Bone 2008; 43: 549-555.

8 Coupaud S, McLean AN, Allan DB. Role of peripheral quantitative computed tomography in identifying disuse osteoporosis in paraplegia. Skeletal Radiol 2009; 38: 989-995.

9 Rittweger J, Gerrits K, Altenburg T, Reeves N, Maganaris CN, de Haan A. Bone adaptation to altered loading after spinal cord injury: a study of bone and muscle strength. J Musculoskelet Neuronal Interact 2006; 6: 269-276.

10 Cappozza RF, Feldman S, Mortarina P, Reina PS, Schiessl H, Rittweger J et al. Structural analysis of the human tibia by tomographic ( $\mathrm{QQCT}$ ) serial scans. J Anat 2010; 216: 470-481.

11 Lanyon LE, Hampson WG, Goodship AE, Shah JS. Bone deformation recorded in vivo from strain gauges attached to the human tibial shaft. Acta Orthop Scand 1975; 46: 256-268.

12 Tami AE, Schaffler MB, Knothe-Tate ML. Probing the tissue to subcellular level structure underlying bone's molecular sieving function. Biorheology 2003; 40 . 577-590.

13 Holzer G, von Skrbensky G, Holzer L, Pichl W. Hip fractures and the contribution of cortical versus trabecular bone to femoral neck strength. J Bone Miner Res 2009; 24: 468-474. 